\title{
VARIABILIDAD DE LA LÍNEA DE COSTA A PARTIR DE IMÁGENES DE SATÉLITE Y SU RELACIÓN CON LA TEXTURA DEL SEDIMENTO
}

\author{
COASTLINE VARIABILITY FROM SATELLITE IMAGERY AND ITS RELATION WITH SEDIMENT TEXTURE
}

\author{
Carlos Cabezas-Rabadán`, Jaime Almonacid-Caballer, Josep E. Pardo-Pascual, Jesús Soriano- \\ González
}

Grupo de Cartografía GeoAmbiental y Teledetección (CGAT), Universitat Politècnica de València, Camino de Vera s/n 46022, València, España.carlos.cabezas.rabadan@gmail.com; jaialca@upvnet.upv.es; jepardo@cgf.upv.es

\begin{abstract}
:
Beaches are natural environments of great interest for our society. They go through remarkable changes run by key factors that are interconnected according to the literature. A better understanding of these parameters, such as sediment texture and shoreline variability, would be of a great interest for coastal monitoring and planning.

Shorelines of all Landsat 8 (OLI) images available over the course of one year have been obtained for determining the variability that has occurred in different Valencian beaches. Likewise, the relation between shoreline variability and sediment texture has been evaluated, showing that beaches with higher variability over the year have smaller sediment texture, which is also related with gentle slopes, and vice versa.
\end{abstract}

The methodology allows obtaining the shoreline variability, a key parameter of beach morphodynamics, in a semiautomatic way. The variability allows developing a gross estimate of beach texture.

Key words: Shoreline Detection, Coastline Variability, Beach Sediment, Sediment Texture, Landsat 8, Remote Sensing

\section{Resumen:}

Las playas son entornos naturales de enorme interés para nuestra sociedad. Estos espacios están sometidos a grandes cambios regidos por factores clave fuertemente interrelacionados según la literatura. Un mayor conocimiento de estos parámetros, como la textura del sedimento o la variabilidad de la línea de costa de las playas resultaría de gran interés para la monitorización y gestión de la costa.

Se han obtenido las líneas de costa de todas las escenas Landsat 8 (OLI) disponibles a lo largo de un año para, a partir de ellas, determinar la variabilidad de diferentes playas valencianas. Asimismo, se ha evaluado la relación existente entre dicha variabilidad y la textura del sedimento de las playas, mostrando que las playas con mayores cambios en la línea de costa a lo largo del año son aquellas con un tamaño de grano menor, asociado a pendientes más suaves y viceversa.

La metodología seguida incluye la obtención de forma semiautomática de la variabilidad de la línea de costa, un parámetro clave de la morfodinámica costera, y a partir de ella la estimación a grandes rasgos de la textura del sedimento de las playas.

Palabras clave: Detección de la línea de costa, Variabilidad de la línea de costa, Sedimento de playa, Textura del sedimento, Landsat 8, Teledetección

\section{Introducción}

Las playas son espacios naturales que proporcionan protección frente al oleaje y temporales, representan un importante recurso socioeconómico (Prodger et al. 2016), y actúan como soporte físico para los organismos que allí habitan.

Las actuaciones antrópicas en la zona costera generan cambios en el sistema, modifican la distribución y las características del sedimento y en consecuencia alteran la morfología de las playas. La textura del sedimento y la anchura de las playas son factores clave para permitir el mantenimiento de sus funciones. Por tanto, resulta de gran interés disponer de información actualizada de ambos elementos para conocer el estado de la playa y plantear una gestión adecuada.

Sin embargo, las características sedimentarias, particularmente las texturales, pueden ser muy variables, tanto espacial como temporalmente (Guillén 2000; Prodger et al. 2016). Obtener datos actualizados de la textura de los sedimentos de las playas resulta costoso al requerir trabajo de campo y análisis, imposibilitando la monitorización a gran escala.

De hecho, son muchos los estudios ya clásicos que señalan el gran dinamismo que presenta la línea de costa como respuesta a los procesos costeros (Carter

"Corresponding Author: Carlos Cabezas-Rabadán: carlos.cabezas.rabadan@gmail.com 
1988). Se presupone una interrelación entre los diferentes elementos morfodinámicos de la playa, como el oleaje incidente (Hansen and Barnard 2009; Reis and Gama 2010), la fuente de suministros del sedimento y la dimensión de los mismos (Carter 1988; Komar 1998; Dean 1991). Asimismo, la relación de la textura con la pendiente de las playas (Dean 1973; Mclean and Kirk 1969, Vellinga 1983,1984), y la morfología (Masselink and Short 1993; Scott et al. 2011) ha sido tratada por muchos autores. Dean (1991) desarrolló la teoría de un perfil de equilibrio de playa en relación a su sedimento, respecto a la que Bodge (1992) propuso un modelo de perfil exponencial. A partir de las conclusiones alcanzadas por estos estudios, es de esperar que una pendiente más elevada vaya asociada a un material más grosero y, a su vez, a una menor variabilidad en la posición de la orilla. Por tanto, si la pendiente se relaciona con la textura y el comportamiento de la línea de costa, conviene analizar en qué manera se relacionan estas últimas entre sí, pudiendo llegar a estimar la textura de las arenas de las playas a partir del comportamiento de la línea de costa.

En los últimos años se ha realizado un importante esfuerzo investigador tratando de determinar de forma eficiente la posición de la línea de costa partiendo de imágenes espaciales de resolución media (Foody et al. 2005; Muslim et al. 2006; Pardo-Pascual et al. 2012; Almonacid-Caballer 2014; Sánchez-García et al. 2015; García-Rubio et al. 2015; Liu et al. 2017), permitiendo obtener una enorme cantidad de información con una elevada frecuencia de actualización. El empleo de estos algoritmos permite registrar los cambios en la posición de la línea de costa con una alta frecuencia a lo largo de un año (Almonacid-Caballer et al. 2016).

El objetivo de este trabajo es obtener la variabilidad intra-anual de la línea de costa extraída de forma automática a partir de imágenes Landsat y evaluar su relación estadística con el tamaño medio del sedimento en la zona de playa.

Se extraerán automáticamente las líneas de costa de playas de la ciudad de Valencia empleando SHOREX. En ellas se han tomado y analizado texturalmente muestras de arena.

\section{2. Área de estudio}

La zona de estudio se encuentra en el Golfo de Valencia, en la costa este de la Península Ibérica (Fig. 1). Incluye las playas de arena localizadas entre la Patacona y el Perelló.

Se trata de una costa micromareal, con una marea astronómica de menos de $20 \mathrm{~cm}$. Sin embargo, el nivel del mar puede cambiar más de $70 \mathrm{~cm}$ cuando diferentes factores meteorológicos tienen lugar. De hecho, la máxima variabilidad del nivel del mar registrado desde 1993 al 2013 por la boya del Puerto de Valencia fue de 1.32 metros (REDMAR 2015). El régimen de oleaje se caracteriza por olas bajas, con una altura significante de oleaje de 0.7 metros de promedio, y unos periodos cortos, con un periodo de pico de ola de $4.2 \mathrm{~s}$, aunque durante las tormentas las olas pueden alcanzar los 5 metros y 15 segundos de periodo (Pardo-Pascual et al. 2014).
Esta área tiene una fuerte deriva litoral que genera transporte hacia el sur. No obstante, el fenómeno ha sido alterado por la construcción de estructuras de ingeniería civil, como el puerto de Valencia, que actúan como trampas de sedimento causando una acumulación de sedimentos en su parte norte y la erosión hacia el sur.

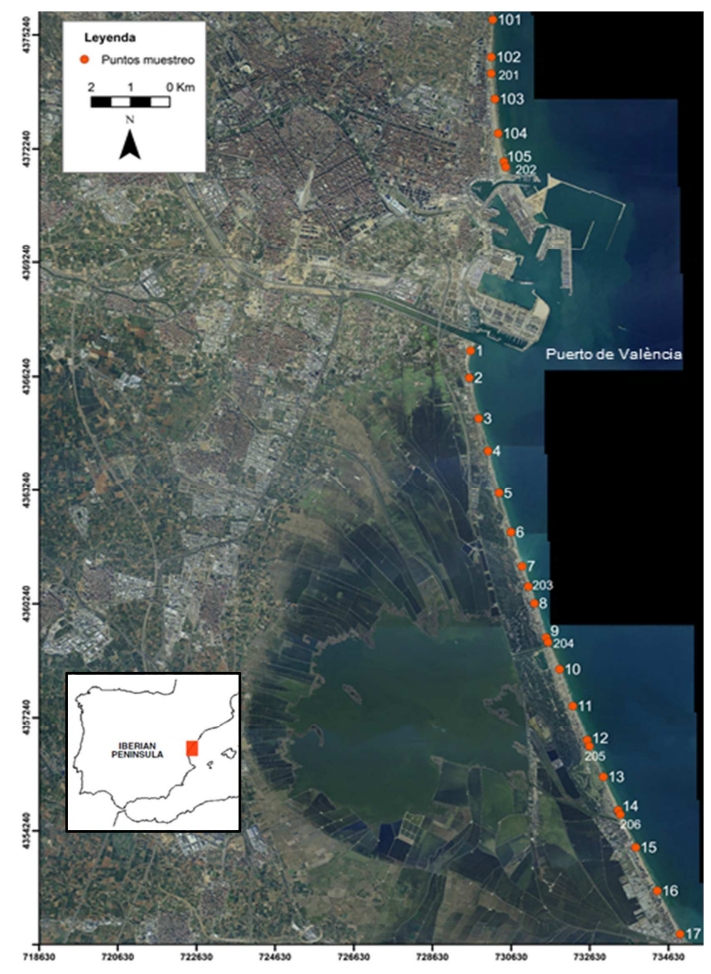

Figura 1: Zona de estudio y puntos de muestreo de sedimento referencia para el cálculo de la variabilidad de la línea de costa (ETRS89 UTM30N).

\section{Metodología}

Se han definido 28 puntos de referencia en las playas, separados entre sí por una distancia cercana a los 1000 metros, tanto al norte como al sur del puerto de Valencia, intentando cubrir una mayor diversidad de tipos de playa. Estas posiciones se tomarán como referencia a la hora de realizar los análisis granulométricos, así como al estudiar los cambios de posición de la línea de costa.

\subsection{Análisis granulométrico}

Se tomaron un total de 56 muestras de arena formando transectos perpendiculares a la línea de costa asociados a los 28 puntos de referencia definidos (Fig. 1). Cada transecto incluye 2 muestras: una en el frente de playa (beach face), donde se produce el swash, y otra en la zona más plana de la playa o estrán (backbeach).

Las muestras fueron recogidas en octubre de 2015, 34 entre la desembocadura del río Túria y la Gola del Perelló (puntos 1-17) y 10 al norte del Puerto de Valencia (101-105), complementadas con otras tomadas en abril de 2015 (201-206). Se tomaron siguiendo un protocolo estandarizado (Sanjaume 1985), retirando siempre los $20 \mathrm{~cm}$ de sedimento más superficiales. El material se secó después en el laboratorio. 
Se tamizaron $50 \mathrm{~g}$ de cada una de las muestras siguiendo la escala Wentworth. Con el programa GRADISTAT (Blott and Pye 2001) se obtuvieron los diferentes índices granulométricos asociados a cada muestra, que permiten analizar cambios en el sedimento (Friedman and Sanders 1978; Batman and Dougherty 1997).

\subsection{Análisis de las líneas de costa}

\subsubsection{Extracción de la posición de la línea de costa}

La posición de línea de costa se obtiene a partir de imágenes registradas por el sensor OLI del satélite Landsat 8 . En concreto se trabaja con la banda 6 , que captura la señal entre los 1566 y $1651 \mathrm{~nm}$ con una resolución espacial de $30 \mathrm{~m}$. Landsat 8 fue lanzado en febrero de 2013, registrando un mismo lugar potencialmente cada 16 días, siendo posible descargar las imágenes de forma gratuita. Para extraer la línea de costa se ha utilizado el programa SHOREX (PardoPascual et al. 2012) que permite obtener automáticamente la línea de costa con precisión subpíxel partiendo de las bandas del infrarrojo de imágenes Landsat. Esta herramienta detecta el borde agua/tierra gracias a la elevada diferencia en la respuesta radiométrica de ambos medios en las bandas infrarrojas. Ajustando una función polinómica se consigue resolver las limitaciones que imponen las grandes dimensiones de pixel. Dado que el algoritmo se basa en la detección de bordes agua-tierra, y no comparando imágenes, no resulta necesario realizar las correcciones radiométricas que son mayormente lineales. Asimismo, se aplica un proceso de georreferenciación también a escala subpixel mediante la correlación cruzada en el espectro de Fourier. Así se alcanza una precisión de 5 metros (error medio cuadrático evaluado sobre escolleras fijas). Cada línea es obtenida en formato puntual (una posición cada $7.5 \mathrm{~m}$ ). Para facilitar la gestión, una vez extraídos y georeferenciados los puntos de la línea de costa se realizó su paso a líneas.

\subsubsection{Imágenes procesadas}

Para contestar a la hipótesis planteada en el presente trabajo se ha realizado la extracción de las líneas de costa disponibles durante el año 2013. Así se ha dispuesto de 16 líneas de costa que cubren la totalidad de la zona estudiada. Se ha trabajado con los puntos de la línea de costa obtenidos de la banda 6 de Landsat 8 , al considerar que es la más estable y que menor error presenta, según ha sido comprobado en trabajos previos (Pardo-Pascual et al. 2012; Almonacid-Caballer 2014).

La primera imagen útil disponible que cubre la zona de estudio en el año 2013 es del 16 de abril, y a partir de entonces la zona del Saler fue capturada 16 veces a lo largo de dicho año (Fig. 2). Esta zona dispone de un mayor número de imágenes al producirse el solape de las escenas 199-33 y 198-33.

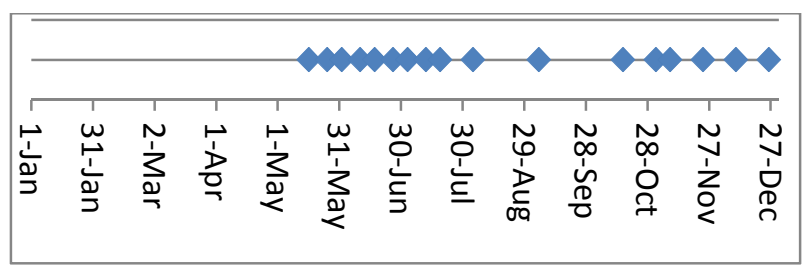

Figura 2: Distribución temporal de las imágenes tomadas por el satélite Landsat 8 sobre el Saler a lo largo del año 2013.

\subsubsection{Determinación de la variabilidad de la línea de costa}

A priori, cuanto menor sea la pendiente de la playa, mayor será el potencial recorrido de la lámina de agua. Una única línea de costa sólo representará una posición instantánea en alguna zona aleatoria de dicho recorrido. Sin embargo, varias líneas de costa, distribuidas adecuadamente, permitirán parametrizar la extensión de dicha lámina. Esto podrá relacionarse con la pendiente u otro parámetro asociado a ella, como en este caso la textura del sedimento. Para ello se han calculado los cambios de posición de la línea de costa en los mismos tramos costeros durante el año 2013 y, posteriormente, se ha obtenido la variabilidad durante ese periodo

Se ha optado por trabajar con segmentos costeros de dos longitudes diferentes: 200 y 400 metros en torno a los puntos de referencia en que se ha realizado el análisis sedimentario (Fig. 1). Se trata de averiguar cuál de ellos señala con mayor claridad la relación entre variabilidad y textura.

Para analizar los cambios en la posición de la línea de costa se ha utilizado el programa Digital Shoreline Analysis System (DSAS) desarrollado por el USGS (Thieler et al. 2009).

En estas zonas se han generado transectos cada 25 metros, sobre los que se han medido las distancias de las diferentes líneas de costa a una línea de referencia común. Con todas las distancias disponibles para los segmentos de análisis de 200 y 400 metros se obtiene una distancia media por fecha. Por tanto, para cada longitud de análisis se dispone de una posición media por fecha, a partir de las cuales se calcula la desviación y el rango a lo largo del año. Estas variables serán consideradas como representativas de la variabilidad anual.

\section{Resultados}

\subsection{Granulometrías}

Las muestras de sedimento, caracterizadas de acuerdo al tamaño medio de sus granos, muestran unos cambios de textura a nivel espacial lógicos a lo largo de los transectos, con un tamaño de sedimento mayor en la rompiente que en el estrán de la playa (Figs. 3 y 4 , Tabla 1).

Longitudinalmente, existen diferencias significativas entre las playas localizadas al norte del puerto de Valencia (101-105, 201 y 202), con un tamaño medio de sedimento cercano a los $200 \mu \mathrm{m}$ para la rompiente y el estrán, y las playas localizadas al sur (1-17, 203-206), donde el sedimento presenta un mayor tamaño. El sedimento más grueso aparece asociado a zonas 
erosivas al sur del puerto, donde el sedimento más fino está siendo lavado.

A nivel temporal también se encuentran variaciones destacables, ya que los muestreos realizados durante la primavera (201-206) muestran valores en ocasiones significativamente diferentes a los correspondientes al mes de octubre sobre la misma localización.

Debemos indicar que, en el caso del punto 2, no se disponen de datos para el interior de la playa ya que se trataba de un escollerado artificial.

\subsection{Variabilidad de la línea de costa}

En la tabla 1 se exponen los valores de granulometría asociados a cada punto de análisis, así como los valores de variabilidad (desviación típica y rango) de las líneas de costa en los segmentos de 200 y 400 metros.

Los valores de rango y desviación estándar son similares y siguen la misma tendencia al analizar los segmentos de 200 y 400 metros. No obstante, puntualmente la desviación estándar y especialmente el rango resultan sustancialmente mayores para los tramos de 200 metros, tal y como ocurre en los puntos 9 y 101.

Se ha de señalar que, debido a su localización geográfica, no se ha podido calcular la variabilidad de los puntos 1, 105 y 202 sobre un tramo de 400 metros, ya que buena parte del análisis hubiese entrado en entornos artificiales como la desembocadura del Túria y el dique norte del puerto de Valencia respectivamente.

Tabla 1: Valores de tamaño medio del sedimento y de cambio anual de la línea de costa a lo largo del 2013 analizando segmentos de 200 y 400 metros alrededor de los puntos de referencia

\begin{tabular}{|c|c|c|c|c|c|c|}
\hline & \multicolumn{2}{|c|}{ Tamaño medio sedimento $(\mu \mathrm{m})$} & \multicolumn{2}{|r|}{$200 \mathrm{~m}$} & \multicolumn{2}{|r|}{$400 \mathrm{~m}$} \\
\hline & Rompiente & Estrán & Rango (m) & Desviación estándar (m) & Rango (m) & Desviación estándar (m) \\
\hline 1 & 262.8 & 198.3 & 24.06 & 7.31 & & \\
\hline 2 & 589.2 & 944.4 & 10.90 & 2.85 & 9.51 & 2.65 \\
\hline 3 & 919.6 & 289.3 & 8.52 & 2.29 & 7.29 & 1.96 \\
\hline 4 & 874.5 & 342.4 & 10.30 & 2.87 & 9.22 & 2.81 \\
\hline 5 & 791.4 & 624.2 & 9.03 & 2.30 & 8.89 & 2.07 \\
\hline 6 & 500.9 & 359.1 & 10.33 & 2.37 & 10.50 & 2.32 \\
\hline 7 & 687.0 & 543.0 & 12.05 & 2.87 & 9.39 & 2.43 \\
\hline 8 & 691.2 & 300.1 & 9.64 & 2.69 & 11.01 & 3.07 \\
\hline 9 & 346.9 & 272.7 & 21.85 & 4.56 & 10.35 & 2.61 \\
\hline 10 & 720.4 & 368.0 & 14.44 & 2.99 & 11.77 & 2.55 \\
\hline 11 & 645.4 & 315.3 & 10.66 & 2.77 & 11.45 & 2.76 \\
\hline 12 & 477.8 & 251.8 & 12.42 & 2.96 & 10.69 & 3.27 \\
\hline 13 & 366.8 & 250.0 & 17.84 & 4.27 & 13.70 & 3.67 \\
\hline 14 & 334.0 & 238.2 & 14.09 & 4.20 & 13.81 & 3.86 \\
\hline 15 & 399.0 & 284.5 & 20.34 & 5.47 & 20.23 & 5.67 \\
\hline 16 & 354.7 & 244.2 & 19.07 & 4.98 & 17.36 & 4.94 \\
\hline 17 & 242.9 & 210.4 & 22.43 & 5.99 & 20.08 & 5.36 \\
\hline 101 & 186.6 & 208.1 & 17.15 & 5.09 & 12.99 & 3.83 \\
\hline 102 & 246.1 & 182.6 & 19.77 & 5.10 & 23.04 & 5.63 \\
\hline 103 & 203.7 & 181.5 & 13.57 & 4.14 & 15.42 & 4.13 \\
\hline 104 & 211.9 & 180.1 & 14.71 & 4.33 & 14.88 & 4.35 \\
\hline 105 & 182.6 & 178.4 & 24.69 & 7.29 & & \\
\hline 201 & 213.8 & 221.7 & 22.50 & 5.77 & 24.18 & 5.79 \\
\hline 202 & 166.9 & 169.0 & 24.83 & 6.86 & & \\
\hline 203 & 542.0 & 270.6 & 10.32 & 2.66 & 10.34 & 2.70 \\
\hline 204 & 319.4 & 229.3 & 12.55 & 3.57 & 15.36 & 3.31 \\
\hline 205 & 276.4 & 303.2 & 11.74 & 3.51 & 12.13 & 2.99 \\
\hline 206 & 263.0 & 227.0 & 15.41 & 4.34 & 14.27 & 3.91 \\
\hline
\end{tabular}




\section{Discusión}

\subsection{Relación entre la variabilidad y la textura del sedimento}

La distribución espacial del tamaño de sedimento parece constatar la existencia de una relación entre la textura y las características morfodinámicas de las playas. De este modo, las muestras de sedimento tomadas al norte del puerto de Valencia presentan un tamaño medio más pequeño (Tabla 1). Se trata de playas acumulativas, anchas y de escasa pendiente en las que, según nuestra hipótesis, la variabilidad de la línea de costa debería ser mayor. Por el contrario, al sur del puerto, especialmente en tramos asociados a los puntos 3-8 y 203, el sedimento es más grueso, asociado a procesos erosivos (Pardo-Pascual et al. 2016). En estas playas se espera una mayor pendiente $y$, en consecuencia, una menor variabilidad de la línea de costa.

Para intentar determinar las relaciones existentes entre la variabilidad de la línea de costa y la textura del sedimento partimos de los diferentes parámetros representativos de la textura de las playas y de su variabilidad, que han sido definidos anteriormente (Tabla 1).

Con el propósito de determinar cuáles son los parámetros más representativos de la variabilidad y de la textura del sedimento, así como la amplitud de análisis más adecuada, se relacionan los diferentes parámetros mediante una función lineal (Tabla 2).

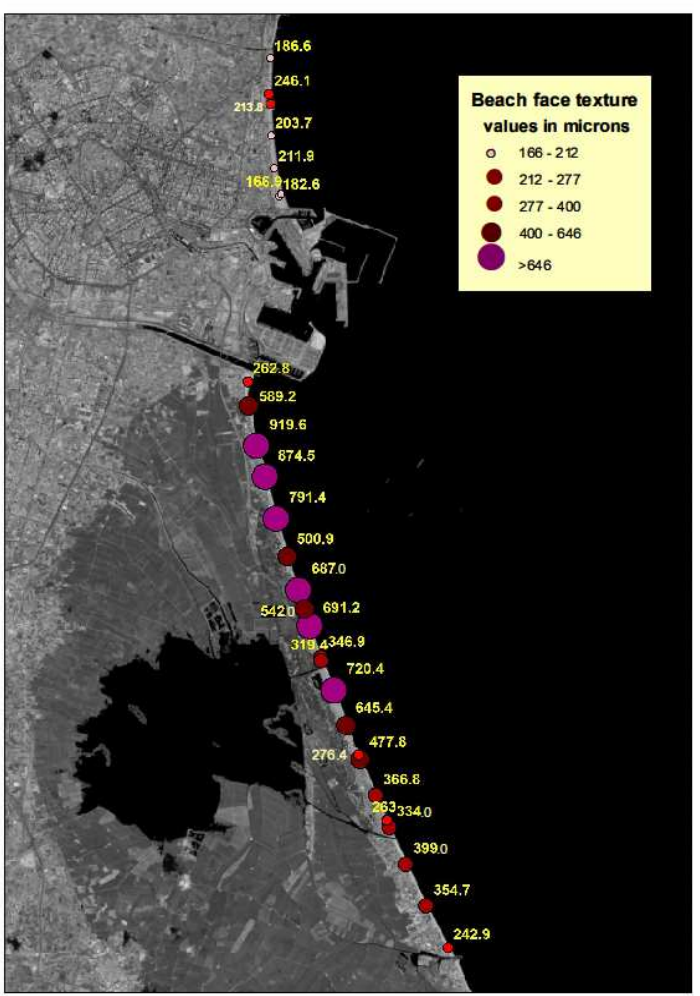

Figura 3: Tamaño medio del sedimento en rompiente $(\mu \mathrm{m})$.

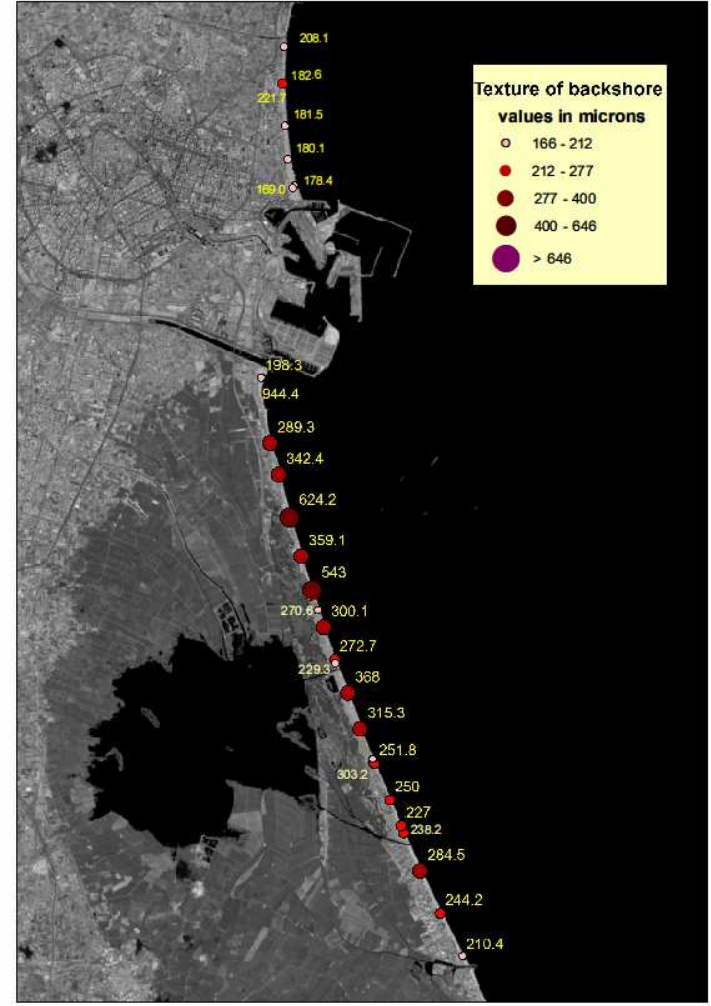

Figura 4: Tamaño medio del sedimento en el estrán $(\mu \mathrm{m})$

Tabla 2: Coeficientes de determinación (R2) del ajuste lineal considerando el rango y la desviación estándar en segmentos de 200 y 400 metros, y la textura en las zonas de rompiente y estrán

\begin{tabular}{c|c|c|c|c} 
& \multicolumn{2}{|c|}{ Desviación estándar } & \multicolumn{2}{c}{ Rango } \\
& $200 m$ & $400 m$ & $200 m$ & $400 m$ \\
\hline Rompiente & 0.596 & 0.500 & 0.517 & 0.485 \\
Estrán & 0.274 & 0.248 & 0.233 & 0.230
\end{tabular}

El mejor ajuste aparece al considerar la desviación estándar de una zona de análisis de 200 metros frente al tamaño medio del sedimento de rompiente (Fig. 5). Estas variables y anchura de análisis parecen ser las que permiten apreciar con mayor claridad la relación entre ambos parámetros, y por tanto son las que consideramos en el resto de análisis.

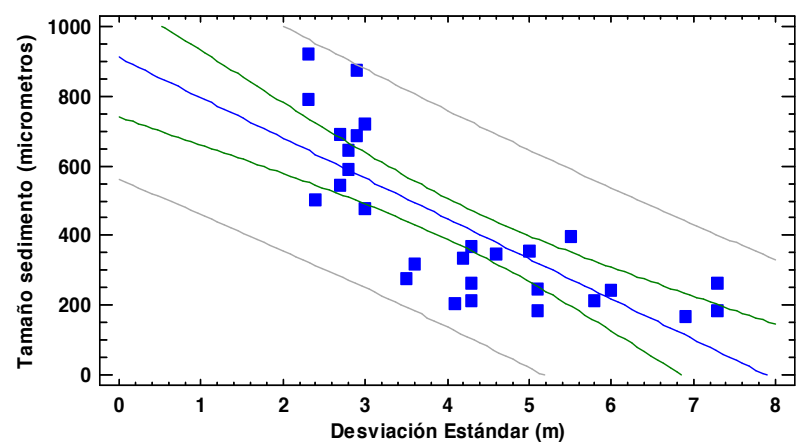

Fig. 5: Ajuste lineal entre el tamaño del sedimento en rompiente, frente a la desviación estándar registrada por la línea de costa, en un entorno de $200 \mathrm{~m}$, junto a los límites de predicción y confianza, con $\mathrm{R}^{2}=0.596$. 
Partiendo de estos mismos datos y empleando un ajuste de segundo grado del tipo $Y=(a+b / X)^{2}$ se alcanza un coeficiente de determinación $\mathrm{R}^{2}=0.75$ (Fig. 6).

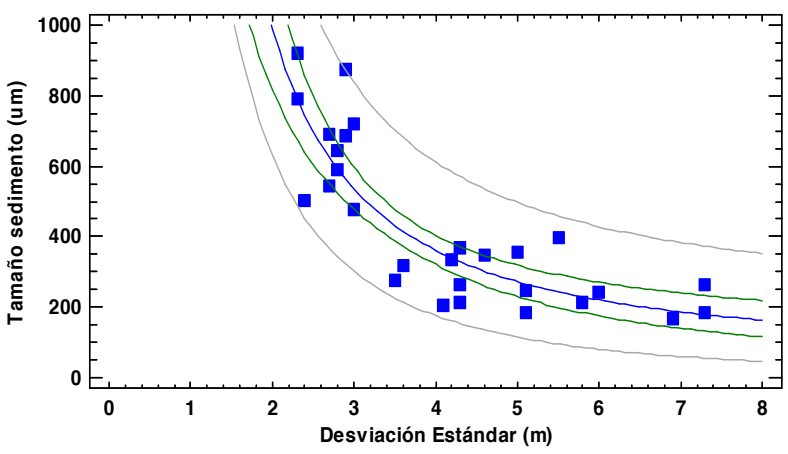

Fig. 6: Ajuste raíz cuadrada de-Y X-inversa entre el tamaño medio del sedimento de rompiente y la variabilidad de la línea de costa expresada como desviación estándar, junto a los límites de predicción y confianza, con $\mathrm{R}^{2}=0.748$.

Este análisis corrobora la idea de que sí existe una relación alta e inversa entre la variabilidad y la textura, pese a que presenta un intervalo de predicción bastante amplio.

Según esta regresión, es posible realizar una estimación grosera de la textura de una playa a partir de la variabilidad de las líneas de costa a lo largo de un año.

Sin embargo, analizando gráficamente los datos disponibles se observan comportamientos aparentemente diferenciados entre dos grupos de playas.

Para ilustrarlo planteamos un análisis clúster, en el que se evidencia la presencia de 2 grupos de playas según el tamaño de su sedimento, con una variabilidad muy diferente (Fig 7).

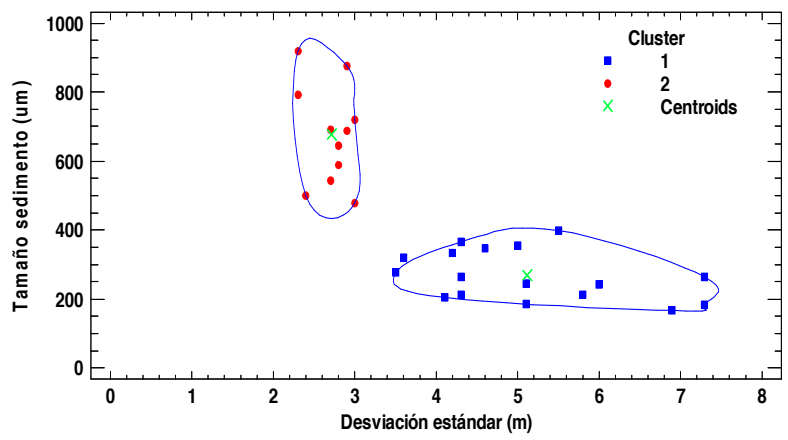

Fig. 7: Análisis de conglomerados con el método de Ward y distancia Euclidea, definiendo 2 clusters y sus centroides.

Aparentemente, a partir de cierto tamaño de sedimento de rompiente, cercano a los $400 \mu \mathrm{m}$, la variabilidad de la línea de costa, expresada como desviación estándar, se mantiene entre $2.5 \sim 3$ metros, mientras que para un tamaño inferior a $400 \mu \mathrm{m}$ la desviación es mucho más alta, y va desde los 3.5 a los 7.5 metros.

La existencia de estos 2 grupos queda respaldada estadísticamente al obtener los mismos clusters empleando diferentes métodos de análisis. Asimismo, para complementar este análisis se realizó un análisis discriminante que clasificó adecuadamente todos los datos disponibles, y en el que las variables más útiles para la clasificación fueron nuevamente la variabilidad en un segmento de 200 metros y el tamaño del sedimento en rompiente.

Ante los datos aportados cabe preguntarse: ¿Existe algún umbral a partir del cual la variabilidad de la playa ya no aumenta a pesar de disminuir la pendiente? ¿Cómo se interrelacionan ambas variables en función de la variable que parece ser su nexo, la pendiente? ¿Hay alguna razón física que explique los grandes incrementos en la variabilidad a partir de cierto tamaño de arena? Nos encontramos ante unas cuestiones a las que no podemos responder con los datos disponibles.

\section{2. $\quad$ Factores que influyen en el análisis}

\subsubsection{Definición de la variabilidad}

La metodología empleada parte de la posición de la línea de costa extraída de diferentes imágenes Landsat. Por limitaciones meteorológicas, éstas se encuentran disponibles en un número limitado a lo largo del año y con una distribución no homogénea Así pues, se dispone de más imágenes en verano que en invierno.

La variabilidad depende de que las líneas instantáneas con que se realiza el cálculo representen adecuadamente el recorrido de la lámina de agua, permitiendo obtener una desviación estándar realmente representativa.

Para este estudio se ha dispuesto de 16 líneas de costa a lo largo de 9 meses. El hecho de que no se encuentren uniformemente distribuidas es una ventaja dado que permite evaluar la variabilidad evitando tendencias temporales, minimizando oleajes y mareas puntuales.

En cualquier caso, la posibilidad de disponer de un mayor número de líneas de costa Landsat, así como la posibilidad de obtenerlas también de imágenes del satélite SENTINEL-2, no hace sino reforzar la utilidad de esta metodología, capaz de gestionar grandes volúmenes de información ambiental.

La obtención de las líneas de costa mediante SHOREX (Pardo-Pascual et al. 2012; Almonacid-Caballer 2014) presenta un error estimado de 5 metros (EMC), evaluado sobre tramos fijos de escollera. Esta incertidumbre inherente al método de deducción de la línea de costa introduce ruido en la definición de la orilla, de forma que las variabilidades aquí analizadas presentan un componente independiente a la propia variabilidad experimentada por la línea de costa.

En cuanto a la longitud de análisis, se ha intentado determinar si la relación existente entre la variabilidad y el tamaño del sedimento aparece con mayor claridad en un segmento de 200 o 400 metros de longitud. Un análisis en un entorno más pequeño corre el riesgo de sobreestimar cambios puntuales en la posición de la orilla con poca relación con la pendiente y textura de todo el sector, como los beach cusps. Por el contrario, al analizar tramos demasiado largos se desdibujan las diferencias entre zonas con diferente naturaleza.

De hecho, los resultados obtenidos muestran que, para nuestro propósito, resulta más adecuado trabajar con tramos de playa de 200 metros. Esto se debe a que el análisis en un segmento corto se ve menos 
condicionado por la mezcla de ambientes heterogéneos, con tamaños de grano y pendientes diferentes.

Para definir la variabilidad se ha propuesto emplear el rango y la desviación estándar intra-anuales. A priori, el rango podría representar mejor la variabilidad de la playa. Sin embargo, dado que contamos con un número limitado de líneas de costa, la desviación estándar parece un indicador más robusto que el rango al ser más resistente a medidas extremas, outliers, etc.

Los resultados muestran en todos los casos analizados una relación más alta entre la textura y la desviación estándar que frente al rango (Tabla 2). Por todo ello la desviación estándar parece el indicador más adecuado, aun cuando se deberá analizar en el futuro si otros indicadores del cambio de posición presentan una mejor relación con la textura del sedimento.

\subsubsection{Definición de la textura}

Respecto al empleo del tamaño medio de los componentes del sedimento como parámetro para su caracterización, pese a que es ampliamente utilizado junto al D50, cabe preguntarse si es representativo de la textura del sedimento en una playa. Diferentes estudios apuntan a que una única variable no siempre es representativa de la naturaleza y comportamiento del sedimento (Holland and Elmore 2008; Medina et al. 1994), especialmente en las playas con una textura mixta de gravas y arenas en las que sedimento presenta una distribución bimodal. El empleo de otros parámetros descriptores de la textura, como diferentes percentiles o la clasificación (sorting), podrían aportar información útil para mejorar la comprensión de la relación entre la textura del sedimento, la pendiente, y la variabilidad de las playas.

Respecto a la representatividad espacial del tamaño de los componentes del sedimento, se ha determinado la textura de las playas a partir del tamaño de grano de rompiente. Esto resulta lógico al tratarse, a priori, de la zona más influenciada por el comportamiento marino, a diferencia del estrán u otras áreas más internas de la playa. Sin embargo, este valor responde a la toma de muestras realizada en un único punto y momento, con la consiguiente limitación de su representatividad. Diferentes estudios apuntan a la existencia de una gran variabilidad espacial y temporal en la textura del sedimento (Prodger et al. 2016; Gujar et al. 2011; Gallagher et al. 2011), que lleva a una redistribución de los diferentes tamaños de grano a lo largo de un perfil de playa (Medina et al. 1994).

En este caso, hemos comprobado que tal variabilidad temporal existe. De hecho, los puntos de muestreo 201 y 206 muestran un cambio de perfil de verano a invierno, con un cambio sustancial en el tamaño entre ambas estaciones (Tabla 1). Esto puede tener relación con un pequeño temporal previo a la campaña de muestreo, que pudo movilizar la arena presente en la playa (especialmente en rompientes, donde mejores interrelaciones se han encontrado).

Por lo tanto, debemos cuestionarnos en qué medida la granulometría varía a lo largo del año, y hasta qué punto la variabilidad de la línea de costa registrada durante todo un año se puede relacionar con la granulometría de un momento concreto. Las conclusiones alcanzadas por Medina et al. (1994) indican que, para un perfil de playa dentro de una misma unidad fisiográfica, la distribución granulométrica a lo largo del perfil, si bien es cambiante, se mantiene constante en su conjunto ("master grain size distribution"). Esto sugiere que sería razonable relacionar la variabilidad de la posición de la línea de costa, no tanto con los valores granulométricos puntuales (de un único lugar e instante determinado), sino con una muestra representativa de la totalidad del sedimento presente a lo largo de un perfil.

Asimismo, respecto a la variabilidad espacial, en esta zona aparecen cambios en el tamaño de grano asociados a la deriva norte-sur que se da en este sector (Sanjaume and Pardo-Pascual 2007). Este fenómeno natural, unido a la presencia de una serie de obstáculos al transporte longitudinal de origen antrópico, genera situaciones de acumulación y erosión a norte y sur de dichas estructuras respectivamente. Estas actuaciones, así como diferentes realimentaciones artificiales de arena y la eliminación de obras de ingeniería civil, han tenido grandes repercusiones sobre las características del sedimento y pueden haber desdibujado parte de los resultados.

\section{Conclusiones}

Los resultados muestran una clara relación entre la textura media de la arena en las playas y la variabilidad de su línea de costa, siendo mayor la variabilidad en playas con un menor tamaño de los componentes del sedimento. Esta relación no es lineal y podría estar motivada por la diferente pendiente que presentan las playas.

La variabilidad ha sido descrita como la desviación estándar del conjunto de líneas de costa observadas a lo largo de un año en distintos segmentos de costa. Dichas líneas de costa han sido obtenidas con un EMC previsible de $5 \mathrm{~m}$ a partir de escenas Landsat procesadas con SHOREX.

Poder dar un valor numérico a la variabilidad de la línea de costa permite relacionarla con los datos de textura tomados en campo. Dicha relación resulta de gran interés dado que permitiría, a partir de observación satelital, estimar grosso modo un parámetro clave en el comportamiento de las playas como es su textura.

Conocer los cambios en las características del sedimento de forma actualizada serviría para la monitorización y detección temprana de cambios en las playas y procesos erosivos, así como para evaluar la efectividad 0 efectos negativos de las actuaciones antrópicas realizadas en ellas. Resulta importante destacar que esta metodología abre las puertas a un análisis retrospectivo aprovechando la serie temporal de las plataformas Landsat.

No obstante, también se observa la existencia de un umbral en el tamaño de los granos del sedimento a partir del cual la variabilidad de las playas se ve muy reducida. Probablemente, este umbral en dicho tamaño de sedimento implica un comportamiento morfodinámico diferente.

En los sistemas costeros intervienen multitud de fenómenos. Su influencia a diferentes escalas temporales y espaciales impide una correcta parametrización del sistema. En este sentido, encontrar buenos descriptores y el desarrollo de nuevas 
metodologías/algoritmos para medirlos pueden llevar nuevas opciones con que describir de manera útil la dinámica costera.

\section{Agradecimientos}

Este proyecto ha sido realizado con la ayuda de la beca FPU15/04501 concedida por el MECD a C. Cabezas, así como con el proyecto RESETOCOAST (CGL201569906-R) del programa Retos del MINECO. Los autores agradecen el apoyo de la Oficina Técnica DevesaAlbufera.

\section{References}

ALMONACID-CABALLER, J., SÁNCHEZ-GARCÍA, E., PARDO-PASCUAL, J.E., BALAGUER-BESER, A.A., and PALOMAR-VÁZQUEZ, J., 2016. Evaluation of annual mean shoreline position deduced from Landsat imagery as a mid-term coastal evolution indicator. Marine Geology, 372, pp. 79-88. https://doi.org/10.1016/j.margeo.2015.12.015.

ALMONACID-CABALLER, J., 2014. Tesis: Detección subpixel de la línea de costa. Universitat Politècnica de València.

BATMAN, S., and DOUGHERTY, E.R., 1997. Size distributions for multivariate morphological granulometries. Optical Engineering, 36(5), pp.1518-1529. https://doi.org/10.1117/1.601340.

BLOTT, S. J., and PYE, K., 2001. Gradistat: A grain size distribution and statistics package for the analysis of unconsolidated sediments. Earth Surface Processes and Landforms, 26(11), pp. 1237-1248. https://doi.org/10.1002/esp.261.

BODGE, K.R., 1992. Representing Equilibrium Beach Profiles with an Exponential Expression. Journal of Coastal Research, 8(1), pp. 47-55. Retrieved from http://journals.fcla.edu/jcr/article/viewFile/78654/76055.

CARTER, B., 1988. Coastal environments : an introduction to the physical, ecological and cultural systems of coastlines.

DEAN, R., 1973. Heuristic models of sand transport in the surf zone. Firt Australian Conference on Coastal Engineering.

DEAN, R., 1991. Equilibrium beach profiles: characteristics and applications. Journal of Coastal Research.

FOODY, G.M., MUSLIM, A.M., and ATKINSON, P.M., 2005. Super-resolution mapping of the waterline from remotely sensed data. International Journal of Remote Sensing, 26:24, pp. 5381-5392. https://doi.org/10.1080/01431160500213292.

FRIEDMAN, G. and SANDERS, 1978. Principles of sedimentology. John Wiley \& Son.

GARCÍA-RUBIO, G., HUNTLEY, D., and RUSSELL, P., 2015. Evaluating shoreline identification using optical satellite images. Marine Geology, 359, pp. 96-105. https://doi.org/10.1016/j.margeo.2014.11.002.

GALLAGHER, E.L., MACMAHAN, J., RENIERS, A.J.H.M., BROWN, J., and THORNTON, E.B., 2011. Grain size variability on a rip-channeled beach. Marine Geology, 287(1), pp. $43-53$. https://doi.org/10.1016/j.margeo.2011.06.010.

GUILLÉN, J., 2000. Variabilidad del tamaño del sedimento en la zona litoral, Geomorfología Litoral. Procesos activos (J.R. Andrés y F.J. Gracia, Editores), pp. 45-66, Instituto Tecnológico GeoMinero de España, Madrid.

GUJAR, A.R., GANESAN, P., IYER, S.D., GAONKAR, S.S., AMBRE, N.V, LOVESON, V.J., and MISLANKAR P.G., 2011. Influence of morphodynamic variability over seasonal beach sediments and its probable effect on coastal development. Ocean and Coastal Management, 54(7), pp. https://doi.org/10.1016/j.ocecoaman.2011.03.007.

HANSEN, J.E., and BARNARD, P.L., 2010. Sub-weekly to interannual variability of a high-energy shoreline. Coastal Engineering, 57(11-12), pp. 959-972. https://doi.org/10.1016/j.coastaleng.2010.05.011.

HOLLAND, K.T., and ELMORE, P.A., 2008. A review of heterogeneous sediments in coastal environments. EarthScience Reviews, 89(3-4), pp. 116-134. https://doi.org/10.1016/j.earscirev.2008.03.003.

KOMAR, P., 1998. Beach processes and sedimentation. Prentice Hall.

LIU, Q., TRINDER, J., and TURNER, I.L., 2017. Automatic super-resolution shoreline change monitoring using Landsat archival data: a case study at Narrabeen-Collaroy Beach, Australia. Journal of Applied Remote Sensing, 11(1), 16036. https://doi.org/10.1117/1.JRS.11.016036.

MASSELINK, G., and SHORT, A.D., 1993. The effect of tide range on beach morphodynamics and morphology: a conceptual beach model. Journal of Coastal Research, 9(3), pp. 785-800. https://doi.org/10.2307/4298129.

MCLEAN, R., and KIRK, R., 1969. Relationships between grain size, size-sorting, and foreshore slope on mixed sandshingle beaches. New Zealand Journal of Geology and.

MEDINA, R., LOSADA, M.A., LOSADA, I.J., and VIDAL, C. 1994. Temporal and spatial relationship between sediment grain size and beach profile. Marine Geol., 118(1963), pp. 195-206.

MUSLIM, A., FOODY, G., and ATKINSON, P., 2006. Localized soft classification for super-resolution mapping of the shoreline. International Journal of. Retrieved from http://www.tandfonline.com/doi/abs/10.1080/01431160500396741. 
PARDO-PASCUAL, J.E., ALMONACID-CABALLER, J., CABEZAS-RABADÁN, C., and SORIANO-GONZÁLEZ, J., 2016. Caracterización de la textura de los sedimentos y evolución de la línea de costa desde Pinedo hasta la gola del Perelló mediante imágenes Landsat (1984-2014). València.

PARDO-PASCUAL, J.E., ALMONACID-CABALLER, J., RUIZ, L.A., PALOMAR-VAZQUEZ, J., and RODRIGOALEMANY, R., 2014. Evaluation of storm impact on sandy beaches of the Gulf of Valencia using Landsat imagery series. Geomorphology, 214, pp. 388-401. https://doi.org/10.1016/j.geomorph.2014.02.020.

PARDO-PASCUAL, J.E., ALMONACID-CABALLER, J., RUIZ, L.A., and PALOMAR-VÁZQUEZ, J., 2012. Automatic extraction of shorelines from Landsat TM and ETM+ multi-temporal images with subpixel precision. Remote Sensing of Environment, 123, 1-11. https://doi.org/10.1016/j.rse.2012.02.024.

PRODGER, S., RUSSELL, P., DAVIDSON, M., MILES, J., and SCOTT, T., 2016. Understanding and predicting the temporal variability of sediment grain size characteristics on high-energy beaches. Marine Geology, 376, pp. 109117. https://doi.org/10.1016/j.margeo.2016.04.003.

REDMAR (Red de Mareógrafos de Puertos del Estado), 2015. Resumen de los parámetros relacionados con el nivel delmar y la marea que afectan a las condiciones de diseño y explotación portuaria. Puerto de Valencia, Dirección Técnica Puertos del Estado (Disponible en http://www.puertos.es/es-es).

REIS, A. H., and GAMA, C., 2010. Sand size versus beachface slope - An explanation based on the Constructal Law. Geomorphology, 114(3), pp. 276-283. https://doi.org/10.1016/j.geomorph.2009.07.008.

SÁNCHEZ-GARCÍA, E., PARDO-PASCUAL, J.E., BALAGUER-BESER, A., and ALMONACID-CABALLER, J., 2015. Analysis of the shoreline position extracted from landsat TM and ETM+ imagery. In International Archives of the Photogrammetry, Remote Sensing and Spatial Information Sciences - ISPRS Archives, Vol. 40, pp. 991-998. https://doi.org/10.5194/isprsarchives-XL-7-W3-991-2015.

SANJAUME, E., 1985. Las costas valencianas: sedimentología y morfología. Retrieved from https://dialnet.unirioja.es/servlet/libro?codigo=163487

SANJAUME, E. and PARDO-PASCUAL, J.E., 2007. Cambios de tendencias recientes en la evolución costera del golfo de Valencia: análisis espaciales y sedimentológicos. "Las nuevas técnicas de información geográfica al servicio de la gestión de zonas costeras: Análisis de la evolución de playas y dunas". 5-11.

SCOTT, T., MASSELINK, G. and RUSSELL, P., 2011. Morphodynamic characteristics and classification of beaches in England and Wales. Marine Geology, 286(1), 1-20. https://doi.org/10.1016/j.margeo.2011.04.004.

THIELER, E., HIMMELSTOSS, E., ZICHICHI, J. and ERGUL, A., 2009. The Digital Shoreline Analysis System (DSAS) version 4.0-an ArcGIS extension for calculating shoreline change. Retrieved from https://pubs.er.usgs.gov/publication/ofr20081278.

VELLINGA, P., 1983. Predictive Computational for Beach and Dune Erosion During Storm Surges. Coastal Structures: 806-819.

VELLINGA, P., 1984. A tentative description of a universal erosion profile for sandy beaches and rock beaches. Coastal Engineering, 8(2), pp. 177-188.

WENTWORTH, C.,1922. A scale of grade and class terms for clastic sediments. The Journal of Geology. 